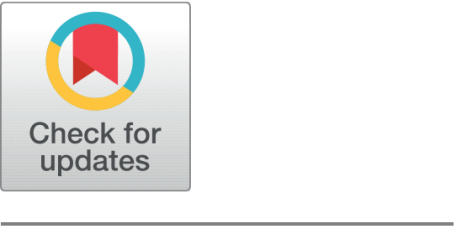

G OPEN ACCESS

Received: 27-03-2020

Accepted: $12-05-2020$

Published: 08-06-2020

Editor: Dr. Natarajan Gajendran

Citation: Nithyanandh S, Jaiganesh V (2020) Quality of service enabled intelligent water drop algorithm based routing protocol for dynamic link failure detection in wireless sensor network. Indian Journal of Science and Technology 13(16): 1641-1647. https://doi.org/ 10.17485/IJST/V13i16.19

*Corresponding author. $S$ Nithyanandh

Ph.D. Scholar, Department of Computer Science, Dr.NGP Arts and Science College, Coimbatore, 641035, Tamil Nadu, India.

Tel.: +91-9750318331

nknithu1@gmail.com

Funding: None

Competing Interests: None

Copyright: $₫ 2020$ Nithyanandh, Jaiganesh. This is an open access article distributed under the terms of the Creative Commons

Attribution License, which permits unrestricted use, distribution, and reproduction in any medium, provided the original author and source are credited.

Published By Indian Society for Education and Environment (iSee)

\section{Quality of service enabled intelligent water drop algorithm based routing protocol for dynamic link failure detection in wireless sensor network}

\author{
S Nithyanandh ${ }^{1 *}, \mathrm{~V}$ Jaiganesh ${ }^{2}$ \\ 1 Ph.D. Scholar, Department of Computer Science, Dr.NGP Arts and Science College, \\ Coimbatore, 641035, Tamil Nadu, India. Tel.: +91-9750318331 \\ 2 Associate Professor, Department of Computer Science, Dr.NGP Arts and Science College, \\ Coimbatore, 641035, Tamil Nadu, India
}

\section{Abstract}

Objectives: To propose a better routing protocol method to increase the lifespan of the network and to make the sensed data to reach from source to destination without any data loss. Methods: This study proposes a routing protocol inspired by nature namely, Quality of Service enabled Intelligent Water Drop Algorithm based Routing Protocol to detect link failures and provide efficient routing. Findings: Comparison has been made with baseline protocols to check its effectiveness and found that the proposed protocol has better performance than the baseline protocols. This research work enhances and adopts the intelligent water drop algorithm with the properties of velocity of water drops and water drops carrying level of soil for finding the better stable route for communication and detecting the link failures dynamically. The results determine that the proposed routing protocol is able to perform better than existing protocols and methods in terms of Energy Depletion rate, Survivability of nodes during transmission, delivering the packets from one end to another end, delay, failure tolerance and increase in Network Lifespan.

Keywords: WSN; Delay; Energy; Routing; Water-Drop

\section{Introduction}

Wireless sensor networks (WSN) need huge number of nodes towards successful communication. In order to minimize the energy utilization, WSN makes use of multi-hop communication rather than single-hop communication. The route between two different sensor nodes is classified as either weak or strong. The route is considered as strong only if sender node and receiver node have more than threshold level of the energy. If the nodes do not have the threshold level of energy, then link may get failed during the transmission of data. Link failures are the major reasons for the WSN to get failed. The present study is to optimize the routing by using the bioinspired protocol to avoid the link failures and to increase the network lifetime. Intelligent Water Drop algorithm is the proposed one to detect the link failure during the data transmission process. 
IWD algorithm is a new optimization method inspired by the water drops flows in rivers which can make optimal paths to the destination.

Two tier optimization protocol ${ }^{(1)}$ proposed to seek the cluster heads, which can enhance the quality of clusters to meet the objective. Routing was performed based on checking the fitness scheme and tree based structure. It attempts to find the best route from cluster head to base stations. Enhanced tree routing ${ }^{(2)}$ proposed for WSN that have structured node based on address assignment methods. It follows the single-hop communication strategy to have better performance. It aimed to reduce the storage and computing cost at each node by using the address assignment method.

$3 \mathrm{D}$ greedy routing ${ }^{(3)}$ was proposed to provide assurance for delivery of data packet to base stations for further processing. To make the data packet to deliver to the destination, it finds the route in 3D instead of 2D. Since greedy method addresses disadvantage, but it has the advantage of providing the packet delivery in reduced time. Balanced routing protocol ${ }^{(4)}$ proposed for WSN utilized in the military for the purpose of tracking and surviving. It works based on batch concept, while performing the routing it segregates the topology into equal sized cluster and designed to operate in a round robin fashion. It developed a rule for handling the data packet by nodes for avoiding the packet loss. Fading aware routing protocol ${ }^{(5)}$ works based on multi-hop communication. It attempts to find the routing path that has no-risk or low-risk. The risk indicates the chance of losing data packet and getting more delay. Before sending the data packet, it analyzes the energy level at each node if the energy found is less than the threshold value then it avoids that route and finds the alternative route.

Energy efficiency based traffic control ${ }^{(6)}$ makes use of deep learning ideas to decrease the consumption of energy. Neural Network was fully utilized to find the optimized path among the available multiple paths, where it checks traffic level each time while sending the packet. Reducing the energy consumption played a major role. Shortcut tree based routing ${ }^{(7)}$ proposed to find the shortest route to destination node based on the distance level. It finds the shortest route based on the tree structure that it maintains at each node. Data packets are not simply sent in all available routes, instead it checks the shortest path. Multicast geographic routing ${ }^{(8)}$ proposed to lessen the energy consumed by nodes for transmitting the data further towards destination. It forwards the data packet only after finding the location of destination node, if data packet is sent by following the routing table it enhances the congestion in WSN. Pareto optimization based routing ${ }^{(9)}$ proposed to find the optimized configuration of the network for ensuring the best routing in WSN. Initially, it finds the available number of cluster heads, members, and quality of available link. It attempts to find the effective routing path that decreases the energy, even the networks are scaled. Geographic cross layer routing ${ }^{(10)}$ attempts to find the better route in disaster relieving operations. It ensembles the medium access control mechanism and handshake mode communication to find the route status. Further, it checks the queue length before sending the data packet in a specific route. Intelligent water drop based coverage-connectivity and lifespan protocol ${ }^{(11)}$ which minimizes energy consumption of the network based routing proposed to reduce the problem of energy holes and maintains the connectivity of networks and coverage ratio of the area. Novel localization ${ }^{(12)}$ proposed the way nonlinear optimization method is used in addition to Received Signal Strength Indicator which is used to determine the interior distance between WSNs nodes. Intelligent water drop algorithm ${ }^{(13)}$ proposed to solve node-selection problem by considering each water drop as an agent which is responsible to find the minimum number of sensor nodes with high data accuracy. Communication protocols for WSNs ${ }^{(14)}$ that gives a useful insight of performance of various protocols in the homogeneous and heterogeneous networks. Enhanced IWDs $^{(15)}$ with exponential and approximate version of simulated annealing algorithm used to solve the multi-depot vehicle routing issues. Few other protocols are proposed ${ }^{(16,17)}$ to deal with feature selection and analyze the performance of the routing to achieve better results. Various researches ${ }^{(18-24)}$ were carried out in this thrust research area, but all focused only on finding the alternate route leading to more delay and packet loss.

Hence, link failures are necessarily to be found before it happens to avoid delay and packet loss. This research work proposes quality of service-enabled nature inspired routing protocol to overcome the link failures that arises dynamically in WSNs and the effect of intelligent water drop algorithm's use for finding the optimal route and detects the node failure. This method falls under the family of swarm intelligence.

\section{Proposed Methodology}

QoS-IWDARP proposed was inspired from nature and swarm optimization algorithms. Two major properties of the proposed protocol are:

1. Water-drops carrying level of soil

2. Water-drops velocity

When QoS-IWDARP flows in the riverbed, the soil is termed as soil (IWD), and the speed is termed as speed $(I W D)$, where the speed gets dynamically changed. When QoS-IWDARP travels from higher-end to lower-end, i.e., from $i$ to $j$, then speed (IWD) 
is increased by $\triangle$ velocity $(I W D)$ value. The level increase is commonly related to the inverse of $i$ and $j$ location soil, which is termed as soil $(i, j)$. The relationship of speed $(I W D)$ and soil $(i, j)$ is possible to mathematically state as:

$$
\triangle \text { speed }(I W D) \propto \frac{1}{\operatorname{soil}(i, j)}
$$

It is possible to formulate a relationship between $\triangle \operatorname{speed}(I W D)$ and $\operatorname{soil}(i, j)$, the speed of IWD is possibly updated by $\operatorname{soil}(i, j)$ as non-linearity based mathematical function and shown in Equation (2):

$$
\triangle v e l^{I W D}(t)=\frac{a_{v}}{b_{v}+c_{v} \times \operatorname{soil}^{2 \alpha}(i, j)}
$$

The parameters $a_{v}, b_{v}, c_{v}$ and $\alpha$ are chosen based on the needs of the user. The extra amount of soil is added to the water-drop is termed as $\triangle \operatorname{soil}(I W D)$ or $\triangle \operatorname{soil}(i, j)$ and an assumption is made that it takes a non-linear time period to travel from $i$ to $j$. The possibility of $\triangle \operatorname{soil}(I W D)$ is mathematically shown in Equation (3)

$$
\triangle \operatorname{soil}(i, j)=\frac{a_{s}}{b_{s}+c_{s} \times \operatorname{time}^{2 \theta}\left(i, j: v^{l} l^{I W D}\right)}
$$

where $\operatorname{time}^{2 \theta}\left(i, j: v e l^{I W D}\right)$ indicates the expected time period for QoS-IWDARP to travel from $i$ to $j$, where it travels with the speed of speed ${ }^{I W D}, \theta, a_{s}, b_{s}$ and $c_{s}$ are positive numbers that are user-defined.

Update Velocity

$$
u v e^{I W D h}(y+1)=u v e^{I W D h(y)+} \frac{b v}{c v+n v \times s o i l^{2(i, j)}}
$$

When QoS-IWDARP pass through the edge of consistency graph, then edge (i,k) utilize Equation (5) and Equation (6) for its update:

$$
\begin{aligned}
& \operatorname{soil}(i, k)=\left\{\begin{array}{l}
(1-\rho L) * \operatorname{soil}(i, k)-\rho l \triangle \operatorname{soil}_{\text {min }} \text { if } \triangle \operatorname{soil}_{(i, k)}<\triangle \operatorname{soil}_{\text {min }} \\
(1-\rho L) * \operatorname{soil}(i, k)-\rho l \triangle \operatorname{soil}_{\text {max }} \text { if } \triangle \operatorname{soil}_{(i, k)}<\triangle \operatorname{soil}_{\text {max }}
\end{array}\right. \\
& (1-\rho L) * \operatorname{soil}(i, k)-\rho l \triangle \operatorname{soil}_{(i, k)} \text { otherwise }
\end{aligned}
$$

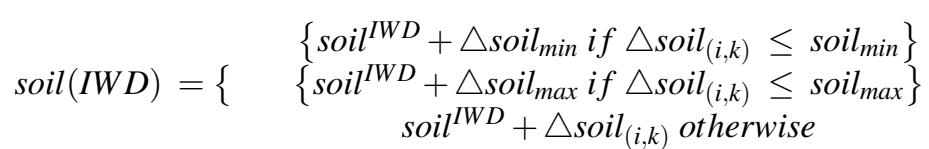

where soil $(I W D)$ represents the soil where $\triangle \operatorname{soil}(i, k)$ holds the value between 0 and 9 , which is used as a parameter to update the local soil. High and low-level values of soil updates are fully dependent on soil present in the edge.

Based on physics simple law of linear motion, $\operatorname{time}\left(i, j: v^{\prime I W D}\right)$ parameter is computed by using speed $(I W D)$ as follows:

$$
\text { time }\left(i, j: v e l^{I W D}\right)=\frac{1}{v e l^{I W D}}
$$

where $\operatorname{soil}(i, j)$ is necessarily made to update because few quantity of soil is eradicated in the path it traveled and it is shown in Equation (8)

$$
\operatorname{soil}(i, j)=\rho_{0} \times \operatorname{soil}(i, j)-\rho_{n} \times \triangle \operatorname{soil}(i, j)
$$

where parameter $\left(\rho_{0}\right.$ and $\left.\rho_{n}\right)$ used for local soil update is necessarily to be a number greater than zero. Amount of soil is computed as

$$
\operatorname{soil}^{I W D}=\operatorname{soil}^{I W D}+\triangle \operatorname{soil}(i, j)
$$

Pareto based investigation is done for performing the calculation towards distance search space. The investigation involves scoring based schedules and for individual schedules, its average mean values are computed. 
QoS-IWDARP gives preference only for the path that has a low level of soil, where it eliminates the path that has a higher level of the soil. It adapts the characteristic of probability-based function, which selects location $j$ that is recommended by the roulette wheel selection method.

$$
p(i, j: I W D)=\frac{f(\operatorname{soil}(i, j))}{\sum_{k \notin v c(I W D)} f(\operatorname{soil}(i, k))}
$$

where $v c(I W D)$ indicates the nodes that are infeasible to the issues ranging from riverbed constraint

$$
f(\operatorname{soil}(i, j))=\frac{1}{\varepsilon_{s}+g(\operatorname{soil}(i, j))}
$$

where $\varepsilon_{s}$ is a positive number that has constant value and prevents division by zero in Eqn.(5.11), and $g(\operatorname{soil}(i, j))$ is a method to put soil only toward positive numbers as shown below

$$
g(\operatorname{soil}(i, j))=\left\{\begin{array}{l}
\operatorname{soil}(i, j) \quad \text { if } \min _{l \notin v c(I W D)} \operatorname{soil}(i, l) \geq 0 \\
\operatorname{soil}(i, j)-\min _{l \notin v c(I W D)} \operatorname{soil}(i, l) \text { otherwise }
\end{array}\right.
$$

The main intention of water-drops is to combine and work to reach the optimum path.

\section{Evaluation of Performance}

Evaluations are conducted to analyze the performance of protocols. Quality of Service of proposed protocol is analyzed against the baseline protocols namely NCCM-DC ${ }^{(12)}$ and EADRA ${ }^{(11)}$. NS2 based simulation is conducted to evaluate the proposed protocol. Simulation settings used for simulation is shown in Table 1. Energy depletion, survivability, packet delivery ratio, delay, failure tolerance and network lifetime are the metrics used for evaluation. The parameter node-count is used for conducting simulation.

\begin{tabular}{ll}
\multicolumn{2}{c}{ Table 1. Simulation Settings } \\
\hline Parameter & Values \\
\hline Nodes Count & $500-2500$ \\
Range of Network & $600 \times 600 \mathrm{~m}^{2}$ \\
Size of Data Packet & $800 \mathrm{bit}$ \\
Initial Transmission Range of Deployed Nodes & $75 \mathrm{~m}$ \\
Initial Energy of Each Node & $20 \mathrm{~J}$ \\
Sensing Range & $10 \mathrm{~m}$ \\
Threshold Distance & $75 \mathrm{~m}$ \\
\hline
\end{tabular}

\section{Results and Discussions}

\subsection{Energy depletion analysis}

This metric shows the speed of energy that gets exhausted at the sending node while data gets transmitted. Figure 1 indicates the proportion of energy depletion at the node count by QoS-IWDARP and previous available protocols EADRA ${ }^{(11)}$ and NCCM$\mathrm{DC}^{(12)}$. It is noticed that QoS-IWDARP have used minimum level of energy and the previous available protocols have used maximum energy level.

\subsection{Node survivability analysis}

Node survivability shows the survival of nodes during the increase in the network load. Figure 2 indicates that nodes getting better survivability in QoS-IWDARP than EADRA ${ }^{(11)}$ and NCCM-DC ${ }^{(12)}$, it is because the energy is saved a lot in QoSIWDARP. 


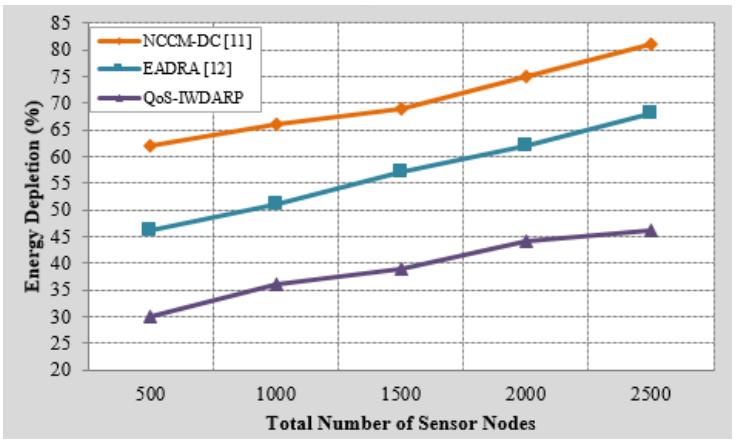

Fig 1. Energy depletion analysis

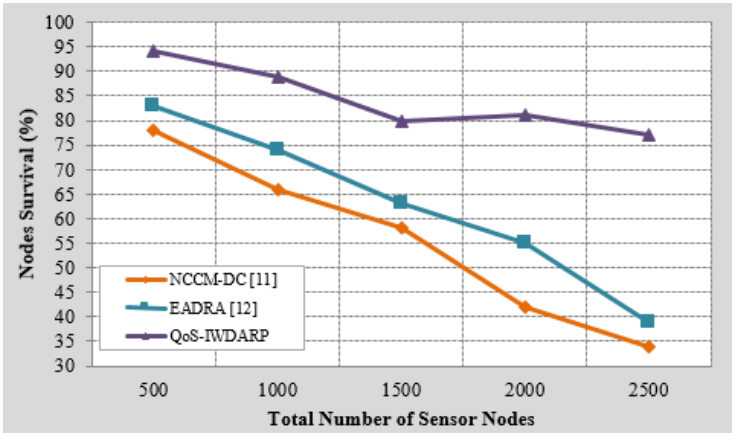

Fig 2. Survivability analysis

\subsection{Packet Delivery Ratio Analysis}

This metric reflects the proportion of receiving the data packets by receiver node in a successful manner. Figure 3 clearly indicates that packet delivery ratio is getting enhanced when node count gets increased, but the previous available protocols EADRA $^{(11)}$ and NCCM-DC ${ }^{(12)}$ didn't deliver the packet in a better manner.

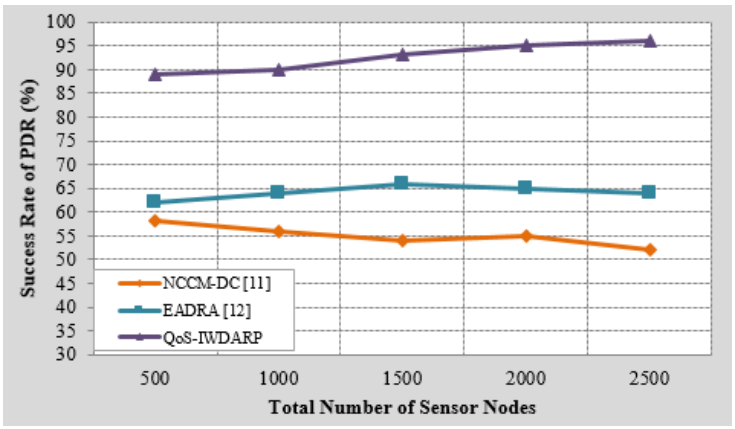

Fig 3. Packet delivery ratio analysis

\subsection{Delay analysis}

This metric shows the consumption of time by the protocols to make the data reach the destination. Figure 4 indicates that QoS-IWDARP consumes minimum time, where EADRA ${ }^{(11)}$ and NCCM-DC ${ }^{(12)}$ takes lengthier time to deliver the data. 


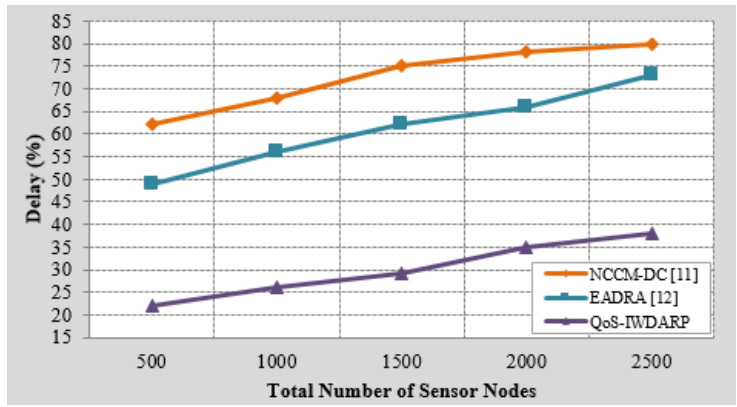

Fig 4. Delay analysis

\subsection{Failure tolerance analysis}

This metric indicates the nodes tolerant rate while the link gets failure. Figure 5 shows nodes tolerance level in QoS-IWDARP, EADRA $^{(11)}$ and NCCM-DC ${ }^{(12)}$. It is very clear that QoS-IWDARP is able to tolerate the link failure by finding the alternate route based on size of the data which is not followed by EADRA ${ }^{(11)}$ and NCCM-DC ${ }^{(12)}$.

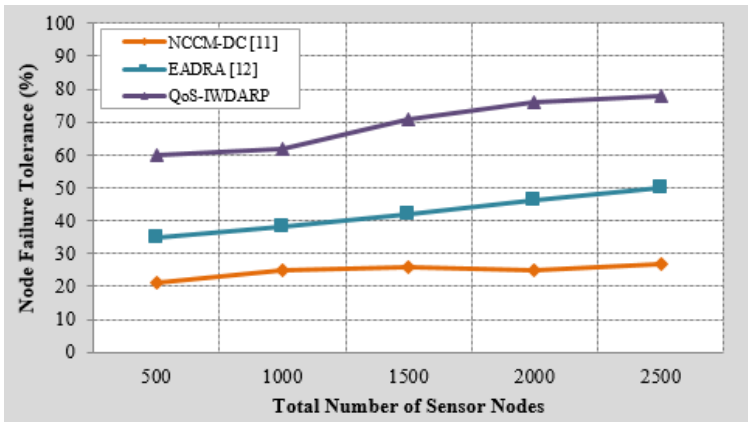

Fig 5. Failure tolerance analysis

\subsection{Network lifetime analysis}

This metric shows successful running of WSN even nodes scaled high. Figure 6 indicates that QoS-IWDARP have outperformed EADRA $^{(11)}$ and NCCM-DC ${ }^{(12)}$ by providing better lifetime to the network.

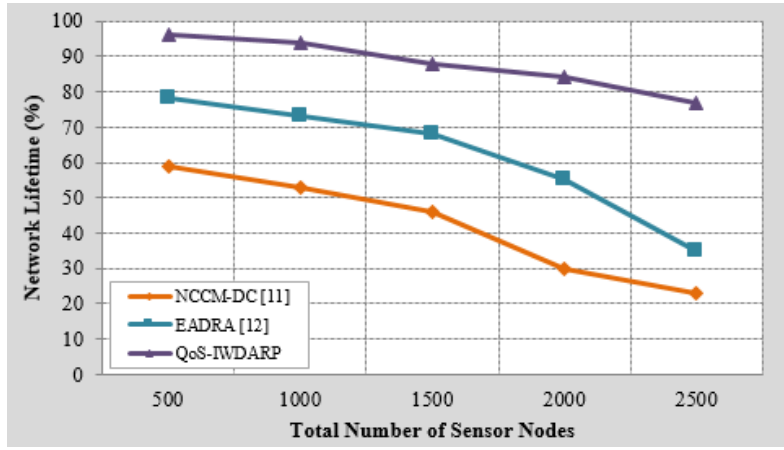

Fig 6. Network lifetime analysis 


\section{Conclusion}

Nature inspired quality of service enabled routing protocol was proposed for WSN to find the better route to destination. Proposed protocol finds the route by analyzing the link failures. During the link failures it finds better alternative route by utilizing the concept of water-drop that flows in different route to reach destination. To analyze the proposed protocol's performance, comparison was performed with previous routing protocols namely Energy Aware Distributing Routing Algorithm (EADRA) and Network-Coding Cluster Level Multi-Path Duty Cycled Protocol (NCCM-DC). The proposed protocol QoS - IWDARP performs better than the previous protocols in seeking the link failures and minimizing the energy consumption to enhance network lifetime that is shown in results graph.

\section{References}

1) Elhabyan RSY, Yagoub MCE. Two-tier particle swarm optimization protocol for clustering and routing in wireless sensor network. Journal of Network and Computer Applications. 2015;52:116-128. doi:10.1016/j.jnca.2015.02.004.

2) Qiu W, Skafidas E, Hao P. Enhanced tree routing for wireless sensor networks. Ad Hoc Networks. 2009;7(3):638-650. doi:10.1016/j.adhoc.2008.07.006.

3) Wang Y, Yi CW, Huang M, Li F. Three-dimensional greedy routing in large-scale random wireless sensor networks. Ad Hoc Networks. 2013;11(4):13311344. doi:10.1016/j.adhoc.2010.10.003.

4) Darabkh KA, El-Yabroudi MZ, El-Mousa AH. BPA-CRP: A balanced power-aware clustering and routing protocol for wireless sensor networks. Ad Hoc Networks. 2019;82:155-171. doi:10.1016/j.adhoc.2018.08.012.

5) Levendovszky J, Tran-Thanh L, Treplan G, Kiss G. Fading-aware reliable and energy efficient routing in wireless sensor networks. Computer Communications. 2010;33:S102-S109. doi:10.1016/j.comcom.2010.07.005.

6) Lu J, Feng L, Yang J, Hassan MM, Alelaiwi A, Humar I. Artificial agent: The fusion of artificial intelligence and a mobile agent for energy-efficient traffic control in wireless sensor networks. Future Generation Computer Systems. 2019;95:45-51. doi:10.1016/j.future.2018.12.024.

7) Wadhwa LK, Deshpande RS, Priye V. Extended shortcut tree routing for ZigBee based wireless sensor network. Ad Hoc Networks. 2016;37(2):295-300. doi:10.1016/j.adhoc.2015.08.025.

8) Huang H, Zhang J, Zhang X, Yi B, Fan Q, Li F. EMGR: Energy-efficient multicast geographic routing in wireless sensor networks. Computer Networks. 2017;129(1):51-63. doi:10.1016/j.comnet.2017.08.011.

9) Elhabyan R, Shi W, St-Hilaire M. A Pareto optimization-based approach to clustering and routing in Wireless Sensor Networks. Journal of Network and Computer Applications. 2018;114:57-69. doi:10.1016/j.jnca.2018.04.005.

10) Niroumand Z, Aghdasi HS. A geographic cross-layer routing adapted for disaster relief operations in wireless sensor networks. Computers \& Electrical Engineering. 2017;64:395-406. doi:10.1016/j.compeleceng.2017.07.021.

11) Sharma R, Lobiyal DK. Intelligent Water Drop Based Coverage-Connectivity and Lifespan Maximization Protocol for Wireless Sensor Networks. Recent Patents on Engineering. 2019;13(3):261-273. doi:10.2174/1872212112666180521082955.

12) Gumaida BF, Luo J. Novel localization algorithm for wireless sensor network based on intelligent water drops. Wireless. 2019;25:597-609.

13) Vajdi A, Zhang G, Wang Y, Zhao Y, Liu D, Wang T. A New Approach Based on Intelligent Water Drops Algorithm for Node Selection in Service-Oriented Wireless Sensor Networks. IEEE Fourth International Conference on Big Data and Cloud Computing. 2014.

14) Ketshabetswe LK, Zungeru AM, Mangwala M, Chuma JM, Sigweni B. Communication protocols for wireless sensor networks: A survey and comparison. Heliyon. 2019;5:e01591-e01591. doi:10.1016/j.heliyon.2019.e01591.

15) Ezugwu AE, Akutsah F, Olusanya MO, Adewumi AO. Enhanced intelligent water drops algorithm for multi-depot vehicle routing problem. PLOS ONE. 2018;13(3):e0193751-e0193751. doi:10.1371/journal.pone.0193751.

16) Alijla BO, Lim CP, Wong LP, Khader AT, Betar MAA. An Ensemble of Intelligent Water Drop Algorithm for Feature Selection Optimization Problem. Applied Soft Computing. 2018;65:531-541. doi:10.1016/j.asoc.2018.02.003.

17) Sayad L, Bouallouche-Medjkoune, \&amp; Aissani L, Djamil. IWDRP: An Intelligent Water Drops Inspired Routing Protocol for Mobile Ad Hoc Networks. Wireless Personal Communications. 2017;94:2561-2581. doi:10.1007/s11277-016-3692-z.

18) Ding $X$, Sun $X$, Huang $C$, Wu. Cluster-level based link redundancy with network coding in duty cycled relay wirelesses sensor network. Computer Networks. 2016;99:15-36. doi:10.1016/j.comnet.2016.02.003.

19) Chanak P, Banerjee I, Sherratt RS. Energy-aware distributed routing algorithm to tolerate network failure in wireless sensor networks. Ad Hoc Networks. 2017;56:158-172. doi:10.1016/j.adhoc.2016.12.006.

20) Nithyanandh S, Jaiganesh V. Dynamic Link Failure Detection using Robust Virus Swarm Routing Protocol in Wireless Sensor Network". International Journal of Recent Technology and Engineering. 2019;8(2):1574-1579. doi:10.35940/ijrte.B2271.078219.

21) J R, R V. Improved frog leap inspired protocol (IFLIP) - for routing in cognitive radio ad hoc networks (CRAHN). World Journal of Engineering. 2018;15(2):306-311. doi:10.1108/wje-08-2017-0260.

22) Ramkumar VJR. "Cuckoo Search Inspired Protocol for Routing in Cognitive Radio Ad Hoc Networks", Computational Intelligence in Data Mining. Advances in Intelligent Systems and Computing. 2017;556:143-156. doi:10.1007/978-981-10-3874-7_14.

23) Nithyanandh S, Jaiganesh V. Reconnaissance Artificial Bee Colony Routing Protocol To Detect Dynamic Link Failure In Wireless Sensor Network". International Journal Of Scientific \& Technology Research. 2019;10(10):3244-3251.

24) Lingaraj M, Prakash A. Power Aware Routing Protocol (PARP) to Reduce Energy Consumption in Wireless Sensor Networks". International Journal of Recent Technology and Engineering. 2019;7(5). Available from: https://www.ijrte.org/wp-content/uploads/papers/v7i5/E1969017519.pdf. 